Figure:

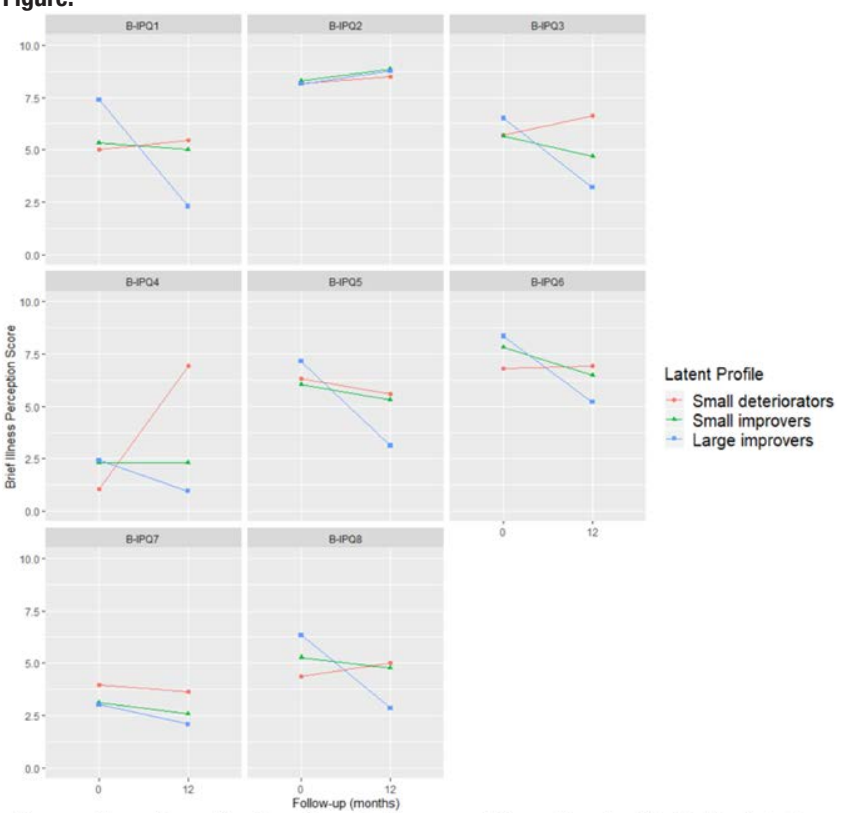

Figure - IIIness Perception Questionnaire items over 12 months, stratified by the latent

profiles [10 represents worst illness perception]

B-IPQ1: How much does arthritis affect your life?

B-IPQ2: How long do you think your arthritis will continue?

B-IPQ3: How much control do you feel you have over your arthritis? (reverse coded)

B-IPQ4: How much do you think your treatment can help your arthritis? (reverse coded)

B-IPQ5: How much do you experience symptoms from your illness?

B-IPQ6: How concerned are you about your arthritis?

B-IPQ7: How well do you feel you understand your arthritis? (reverse coded)

B-IPQ8: How much does your arthritis affect you emotionally?

Disclosure of Interests: James Gwinnutt Grant/research support from: BMS, Sam Norton: None declared, Kimme Hyrich Grant/research support from: Pfizer, UCB, BMS, Speakers bureau: Abbvie, Mark Lunt: None declared, Anne Barton Consultant of: AbbVie, Lis Cordingley Grant/research support from: Unrestricted award from Pfizer unrelated to current abstract, Speakers bureau: Janssen, AbbVie, Celgene, Sanofi, Eli Lilly, Novartis all unrelated to current abstract, Suzanne Verstappen Grant/research support from: BMS, Consultant of: Celltrion, Speakers bureau: Pfizer

DOI: 10.1136/annrhveumdis-2020-eular.1960

\section{SAT0039 ADAPTIVE DEEP LEARNING FOR THE PREDICTION OF INDIVIDUAL DISEASE ACTIVITY IN PATIENTS WITH RHEUMATOID ARTHRITIS}

M. Hügle ${ }^{1}$, G. Kalweit ${ }^{1}$, U. Walker ${ }^{2}$, A. Finckh ${ }^{3}$, R. Muller ${ }^{4}$, A. Scherer ${ }^{5}$, J. Boedecker ${ }^{1}$, T. Hügle ${ }^{6}$ on behalf of the participating physicians of the Swiss Clinical Quality Management in Rheumatic Diseases. ${ }^{1}$ Department of Computer Science, University of Freiburg, Germany, Freiburg, Germany; ${ }^{2}$ Department of Rheumatology, University Hospital Basel, Switzerland, Basel, Switzerland; ${ }^{3}$ Division of Rheumatology, University Hospital Geneva, Switzerland, Geneva, Switzerland; ${ }^{4}$ Department of Rheumatology, Kantonsspital Aarau, Switzerland, Aarau, Switzerland: ${ }^{5}$ SCQM Foundation, Zürich, Switzerland, Zürich,

Switzerland; ${ }^{6}$ Department of Rheumatology, Lausanne University Hospital, CHUV, Switzerland, Lausanne, Switzerland

Background: Rheumatoid arthritis (RA) lacks reliable biomarkers that predict disease evolution on an individual basis, potentially leading to over- and undertreatment. Deep neural networks learn from former experiences on a large scale and can be used to predict future events as a potential tool for personalized clinical assistance.

Objectives: To investigate deep learning for the prediction of individual disease activity in RA.

Methods: Demographic and disease characteristics from over 9500 patients with 65.000 visits from the Swiss Quality Management (SCQM) database were used to train and evaluate an adaptive recurrent neural network (AdaptiveNet). Patient and disease characteristics along with clinical and patient reported outcomes, laboratory values and medication were used as input features. DAS28BSR was used to predict active disease and future numeric individual disease activity by classification and regression, respectively.

Results: AdaptiveNet predicted active disease defined as DAS28-BSR $>2.6$ at the next visit, with an overall accuracy of $75.6 \%$ and a sensitivity and specificity of $84.2 \%$ and $61.5 \%$, respectively. Apart from DAS28-BSR, the most influential characteristics to predict disease activity were joint pain, disease duration, age and medication. Longer disease duration, age $>50$ or antibody positivity marginally improved prediction performance. Regression allowed forecasting individual DAS28-BSR values with a mean squared error of 0.9

Conclusion: Deep neural networks have the capacity to predict individual disease outcome in RA. Low specificity remains challenging and might benefit from alternative input data or outcome targets.

References:

[1] Hügle M, Kalweit G, Hügle T, Boedecker J. A Dynamic Deep Neural Network For Multimodal Clinical Data Analysis. Be Publ Stud Comput Intell Springer Verl. 2020.
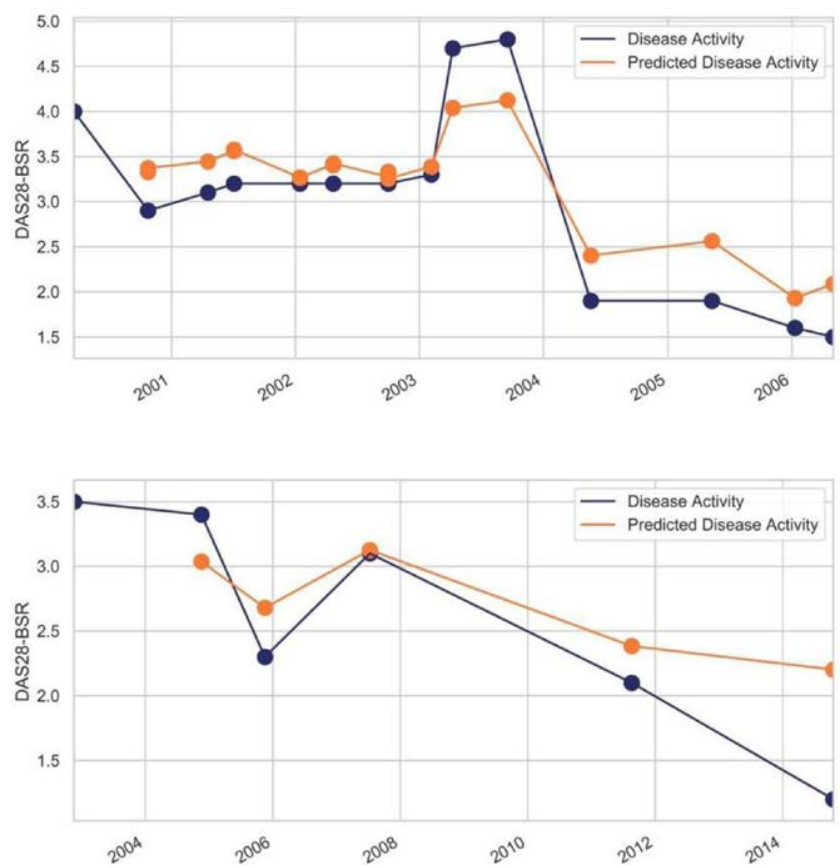

Figure 1. Examples of true disease activity and corresponding predictions of AdaptiveNet by regression analysis. Predictions are made step to step from the current to next visit.

Disclosure of Interests: Maria Hügle Paid instructor for: Lilly, Gabriel Kalweit: None declared, Ulrich Walker Grant/research support from: Ulrich Walker has received an unrestricted research grant from Abbvie, Consultant of: Ulrich Walker has act as a consultant for Abbvie, Actelion, Boehringer Ingelheim, Bristol-Myers Squibb, Celgene, MSD, Novartis, Pfizer, Phadia, Roche, Sandoz, Sanofi, and ThermoFisher, Paid instructor for: Abbvie, Novartis, and Roche, Speakers bureau: Abbvie, Actelion, Bristol-Myers Squibb, Celgene, MSD, Novartis, Pfizer, Phadia, Roche, Sandoz, and ThermoFisher, Axel Finckh Grant/research support from: Pfizer: Unrestricted research grant, Eli-Lilly: Unrestricted research grant, Consultant of: Sanofi, AB2BIO, Abbvie, Pfizer, MSD, Speakers bureau: Sanofi, Pfizer, Roche, Thermo Fisher Scientific, Rudiger Muller Consultant of: AbbVie, Nordic, Sandoz, Almut Scherer: None declared, Joschka Boedecker: None declared, Thomas Hügle Grant/research support from: Abbvie, Novartis, Consultant of: Abbvie, Pfizer, Novartis, Roche, Lilly, BMS DOI: 10.1136/annrheumdis-2020-eular.2712

\section{SAT0040 RISK FACTORS FOR DEVELOPING AND MORTALITY FOR ACUTE EXACERBATION OF RHEUMATOID ARTHRITIS-ASSOCIATED INTERSTITIAL LUNG DISEASE}

S. Izuka ${ }^{1}$, H. Yamashita ${ }^{1}$, Y. Takahashi ${ }^{1}$, H. Kaneko ${ }^{1} .{ }^{1}$ Center Hospital of the National Center for Global Health and Medicine, Shinjuku City, Japan

Background: Among collagen vascular diseases, rheumatoid arthritis-associated interstitial lung disease (RA-ILD) is the most commonly associated with ILD with acute exacerbation (AE) [1]. One study reported that ILD diagnosis at an older age, the usual interstitial pneumonia (UIP) pattern on high-resolution computed tomography, and methotrexate (MTX) use were associated with AE in patients with RA-ILD [2]. However, because these studies included few patients, the risk factors and prognosis of $A E$ in patients with RA-ILD remain unclear 